\title{
Giant Intrathoracic Left-Sided Vagal Schwannoma
}

\author{
${ }^{1}$ Department of Surgery, University Hospital, Marburg, Germany \\ 2 Department of Neurosurgery, Marburg University Hospital, Marburg, \\ Germany \\ ${ }^{3}$ Department of Pathology, Marburg University Hospital, Marburg, \\ Germany \\ ${ }^{4}$ Department of Surgery, Marburg University Hospital, Marburg, \\ Germany
}

Andreas Kirschbaum ${ }^{1} \quad$ Rainer Ritz ${ }^{2} \quad$ Anika Pehl $^{3} \quad$ Detlef Bartsch ${ }^{4}$

Address for correspondence Andreas Kirschbaum, MD, Department of Surgery, University Hospital, Baldingerstrasse, Marburg 35033, Germany (e-mail: Kirschbaum001@gmx.de).

Thorac Cardiovasc Surg Rep 2013;2:19-22.

\begin{abstract}
Extensive intrathoracic tumors are rarely diagnosed radiologically without pre-existing symptoms. If located in the posterior mediastinum, it is most probably a neurogenic tumor. Schwannoma is the most frequent neurogenic neoplasia in this location, and most schwannomas are benign. To specify the diagnosis, a thoracic computed tomography must be done; if the growth is close to the medullary canal, a magnetic resonance tomography of the spinal column is necessary to detect neuroforamen infiltration. Our surgical goal was complete excision of the tumor, although many

Keywords

- chest wall

- mediastinal tumor

- lung

- benign or congenital lesions authors favor a minimally invasive approach. In our patient we performed open, en bloc removal of the tumor; removal of parts of the intraforamen was also necessary, which necessitated revision of the affected neuroforamen. Histologically this was a very rare case of vagal schwannoma (which has an incidence of less than $6 \%$ of all neurogenic tumors). This patient has a very promising prognosis following complete tumor resection.
\end{abstract}

\section{Introduction}

Even large tumors in the posterior mediastinum can remain asymptomatic for a long time provided proximal structures are not compromised. If a large mass is conspicuous in a routine X-ray, the next step is to perform a thoracic computed tomography (CT), which is better suited for assessing the tumor's position and extent. The most common tumors located in the posterior mediastinum are neurogenic, such as neurilemmomas and schwannomas, but the differential diagnosis must also keep tumors of hematopoietic origin and pleural fibromas in mind. Neurogenic tumors represent approximately $20 \%$ of all mediastinal neoplasms in adults. ${ }^{1}$ The definitive diagnosis requires an ultrasound-guided tumor puncture. We describe a rare case of giant schwannoma that most likely originated in the vagus nerve, with an incidence lower than $6 \%{ }^{2}$

\section{Case Report}

This 35-year-old male presented to his general practitioner complaining of a mild cough following a bout of the common flu. Auscultation revealed a left basal impairment; in the absence of any other clinical symptoms, a thoracic X-ray was done to rule out pleuritis. It displayed a surprisingly large well-circumscribed growth measuring $12 \times 20 \mathrm{~cm}^{2}$ in the left center ( - Fig. 1), at this point the patient was admitted to our clinic for further diagnostics. The thoracic CT revealed a smooth, well-circumscribed neoplasia originating in the mid-posterior mediastinum that did not appear to infiltrate. Most of the left lung was totally compromised by the tumor that extended to the neuroforamen. Further preoperative diagnostics entailed a magnetic resonance tomography of the spinal column, as we suspected intraspinal involvement (-Figs. 2 and 3). To assess the tumor's histology we received

December 17, 2012

accepted

January 18, 2013

published online

March 26, 2013
DOI http://dx.doi.org/

10.1055/s-0033-1337368. ISSN 2194-7635.
License terms

(1) (1) $\Theta \circledast$ 

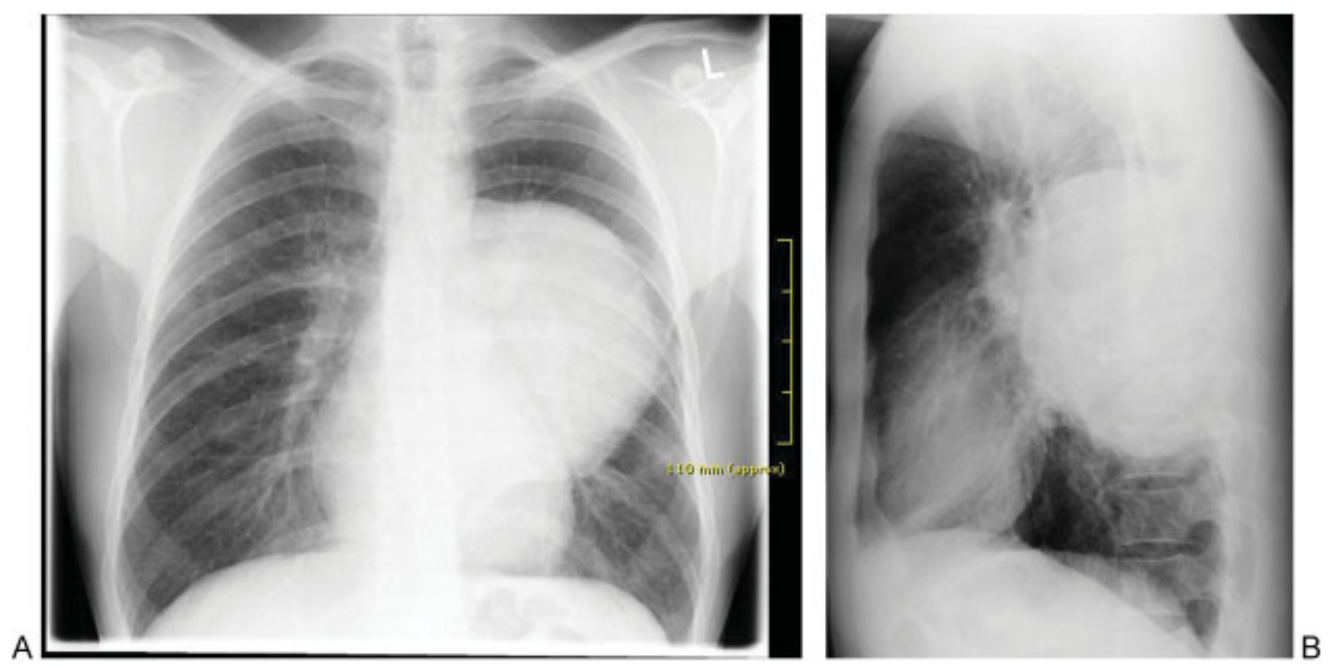

Fig. 1 Thoracic X-ray on two planes.

performed a sonographically guided fine-needle aspiration that showed a benign schwannoma. The only therapy option was radical excision of the tumor; we performed an open surgery via an anterolateral thoracotomy because of the tumor's enormity. On opening the chest wall we observed an enormous paraspinal tumor of very firm and dense consistency. The lung attached to the tumor could be lifted off the tumor, and there were no signs of tumor infiltration (-Fig. 4). The tumor could be moved outside the pleura, and we noticed that it was vascularized via a broad, periaortic bridge of tissue. Some of the vessels in that region (of 5 to $7 \mathrm{~mm}$ in diameter) had to be closed. We were able to remove the tumor en bloc while preserving the vagus nerve that appeared in cranial continuity with the neoplasia (-Fig. 5).

For safety reasons, using a surgical microscope we inspected the neuroforamen, into which the tumor extended in the thorax (extending into the outer third of the neuroforamen). After performing local hemostasis, the left lung was reventilated, whereby the lower left lobe expanded completely after an initial delay. We performed two thorax drainages before closing the thorax (Chapter 24). As the patient required a moderate number of transfusions and as the re-CT revealed an accumulation of fibrin clots accompanied by complete

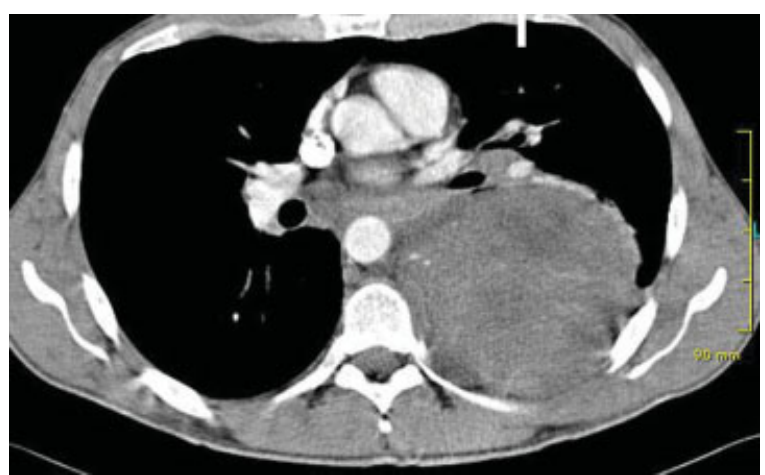

Fig. 2 Computed tomography scan of the thorax. atelectasis of the lower left lobe, we performed a re-thoracotomy on postoperative day 4 that revealed a substantial amount of secretion that we had to endoscopically evacuate from the lower left lobe. There were no indications of active hemorrhage. The patient's further course was uneventful, and he presented no postoperative neurological deficits whatsoever. Histological examination of the tumor revealed a benign schwannoma (with a low mitotic rate) (-Fig. 6). Our patient was released from the hospital on day 10 after the surgery entirely free of symptoms. He has a very good prognosis. He will be coming once a year for follow-up examination.

\section{Discussion}

Neurogenic tumors are the most common type of neoplasia in the posterior mediastinum. This tumor group consists of nerve sheath neoplasias (schwannomas and neural fibromas), tumors of the sympathetic chain (neuroblastomas, ganglion neuroblastomas, and ganglion neurilemmomas), and paragangliomas. Benign schwannomas are the most common neurogenic tumors. They originate in the Schwann cells in the nerve sheath. Histology very seldom reveals mitosis. The very rare cases of malignancy display extensive fusiform masses of pleomorphic spindle cells with necrotic and hemorrhagic zones. As was the case with our patient, schwannomas are asymptomatic for a long period and tend to be discovered by chance. Symptoms (i.e., Horner syndrome) do not occur until surrounding structures have been compromised by the tumor's spread. X-rays generally show a sharply demarcated, round or lobulated growth. ${ }^{3}$ To determine the tumor's extent and resect-ability, a CT of the thoracic spine using a contrast medium must be done. If infiltration of the neuroforamen is suspected (which occurs in 10\% of such cases), a magnetic resonance tomography of the spinal column is also mandatory. Other tumors must be considered in the differential diagnosis, such as extramedullary hematopoietic tumors and pleural fibromas, for which an ultrasound-guided puncture is necessary (that is how we made our patient's differential diagnosis). 

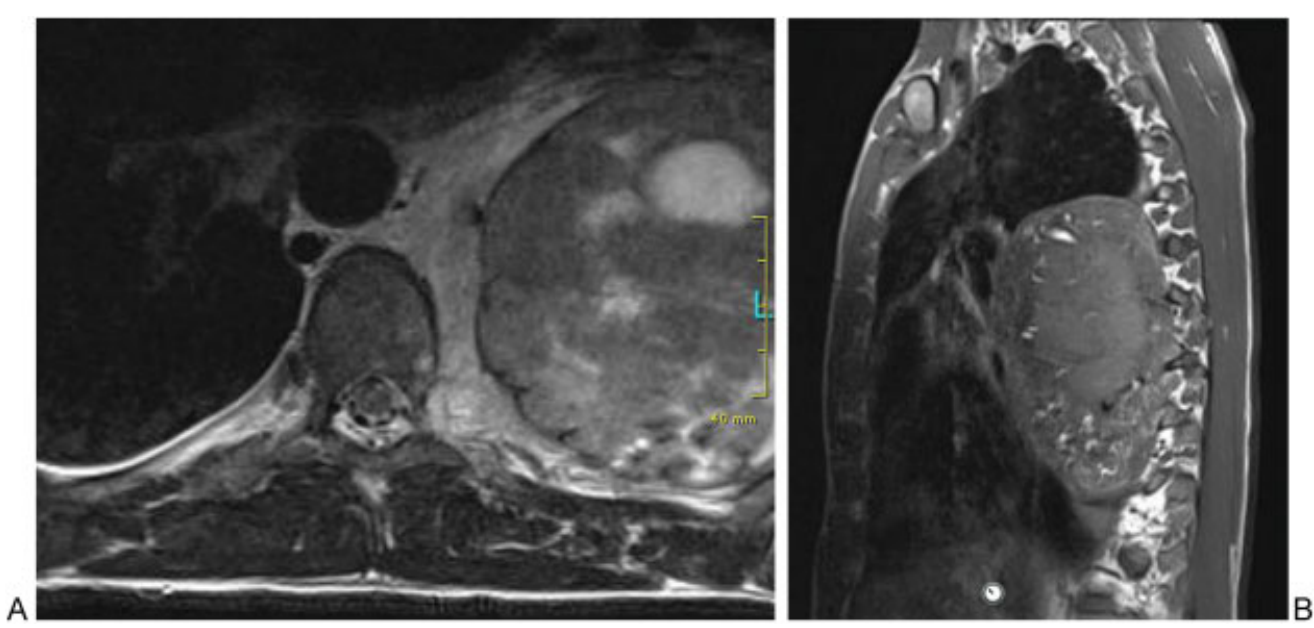

Fig. 3 Magnetic resonance tomography scan of the thoracic spinal column, spinal canal.

Our therapeutic aim was complete removal of the tumor leaving healthy tissue intact. To that end we had to identify the tumor's exact dimensions and extent. We decided on a primarily thoracic approach that enabled us to inspect the neuroforamen. Many authors prefer video-thoracoscopic resection of such tumors. ${ }^{4}$ However, because of this tumor's enormity and improved perspective, we opted for open surgery via an anterolateral thoracotomy. (We believe there are no additional advantages associated with a posterolateral thoracotomy.) Our decision turned out to be correct, as this tumor was quite firmly attached to the chest wall in spite of being morphologically so well circumscribed (in the $\mathrm{CT}$ ) and because of the primarily extrapleural preparation. Moreover, this neoplasia was vascularized periaortically via a broad

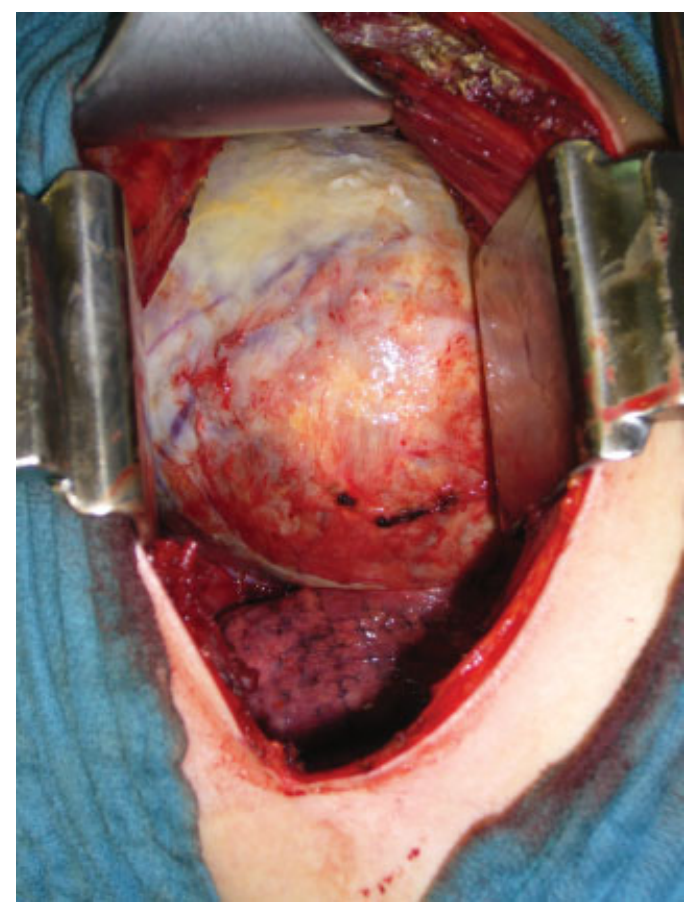

Fig. 4 Intraoperative view of the tumor location and compromised lung. soft-tissue bridge originating in the posterior mediastinum. Here, we had to close several thick neovessels. The tumor extended up to the neuroforamen on the seventh rib but did not infiltrate it. In such a situation, the surgeon's ability to

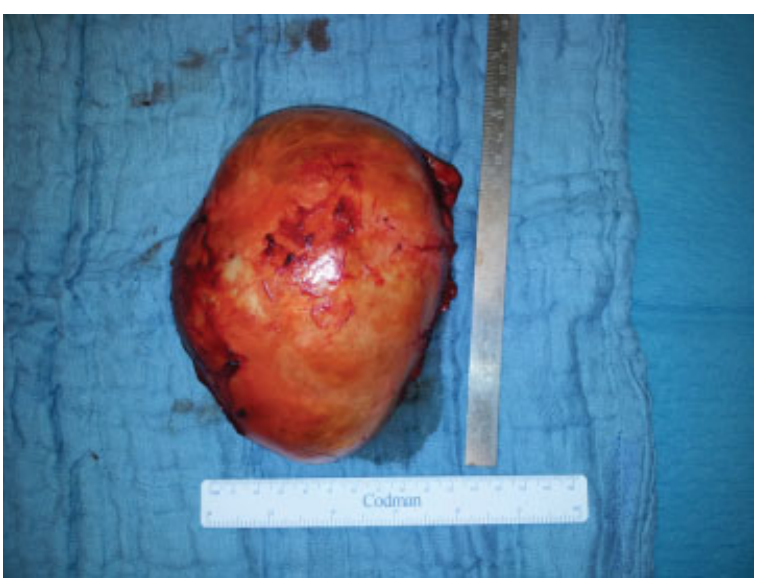

Fig. 5 Resected tumor.

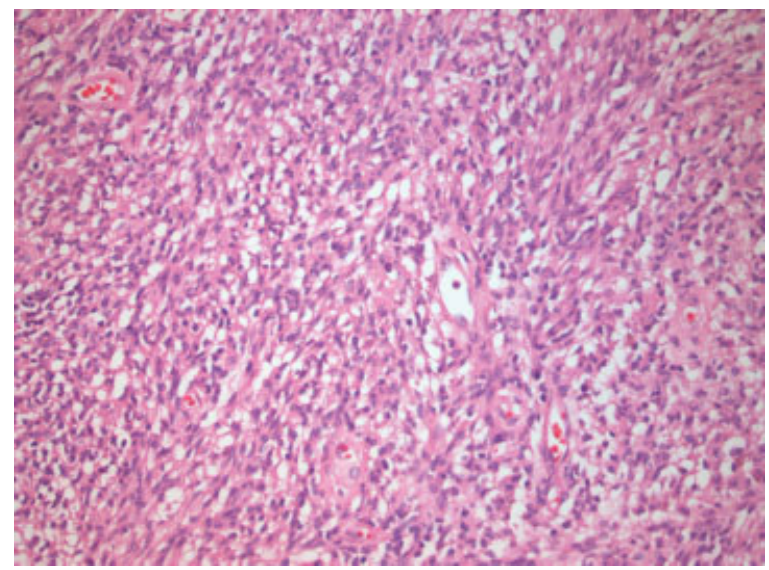

Fig. 6 Hemotoxylin and eosin staining of the tumor: a benign schwannoma (magnification $\times 10$ ). 
finely manipulate and inspect the neuroforamen (using a surgical microscope) is a major advantage, which in turn benefits the patient. ${ }^{5}$

As the left vagus nerve passed through the tumor mass, we were dealing here with a very rare vagus nerve schwannoma. Only between 4 and $6 \%$ of the neurogenic tumors originate in the visceral compartment from the nerve sheaths of the vagus or phrenic nerve. ${ }^{1}$ The left side is more often affected, as the intrathoracic vagus nerve is thicker on that side. ${ }^{6}$

Histologically speaking, schwannomas consist of spindle cells organized in small fascicles with bipolar-oriented or wavy nuclei and long cell appendages. In the Antoni type A growth pattern, the cells are arranged in compact bundles, some in the shape of Verocay bodies (alternating in palisaded nuclei and cell fingers). In the Antoni B areas, the fingers of the rather star-shaped tumor cells form a loose web. Individual large, irregularly shaped, and hyperchromatic nuclei and extensive necrosis, hyalinization, and focal calcification are signs of degenerative changes (referred to as ancient schwannoma)these must not be erroneously considered signs of malignancy. ${ }^{7}$

The successful radical resection of the tumor in our patient has given him a very good prognosis. No adjuvant chemo- therapy was necessary. Nevertheless, we have encouraged him to come for annual checkups entailing a clinical examination and thoracic CT.

\section{References}

1 Davis CJ, Butchart EG, Gibbs AR. Neurilemmoma of the intrathoracic vagus nerve. Eur Respir J 1991;4(4):508-510

2 Rammos KS, Rammos SK, Foroulis CN, Zaramboukas TK. Schwannoma of the vagus nerve, a rare middle mediastinal neurogenic tumor: case report. J Cardiothorac Surg 2009;4:68-71

3 Heitmiller RF, Labs JD, Lipsett PA. Vagal schwannoma. Ann Thorac Surg 1990;50(5):811-813

4 Ayub S, Shakoor MT, Hasan S, Khan JA. Mediastinal mass diagnosed as a benign schwannoma. Singapore Med J 2011;52(9):e167-e169

5 Lan ZG, Chen HF, Huang SQ, Pu Q. One - stage removal of a giant thoracic paraspinal schwannoma via modified hemilaminecotmy and posterolateral thoracotomy. Spine J 2012;12(6):e6-e11. (epub ahead of print, doi:10.1016/j.spine.2012.05.014).

6 Strickland B, Wolverson MK. Intrathoracic vagus nerve tumours. Thorax 1974;29(2):215-222

7 Huang TW, Yang MH, Cheng YL, Tsai WC, Lee SC. Vagus nerve Schwannoma in the middle mediastiunm. Thorac Cardiovasc Surg 2010;58(5):312-314 